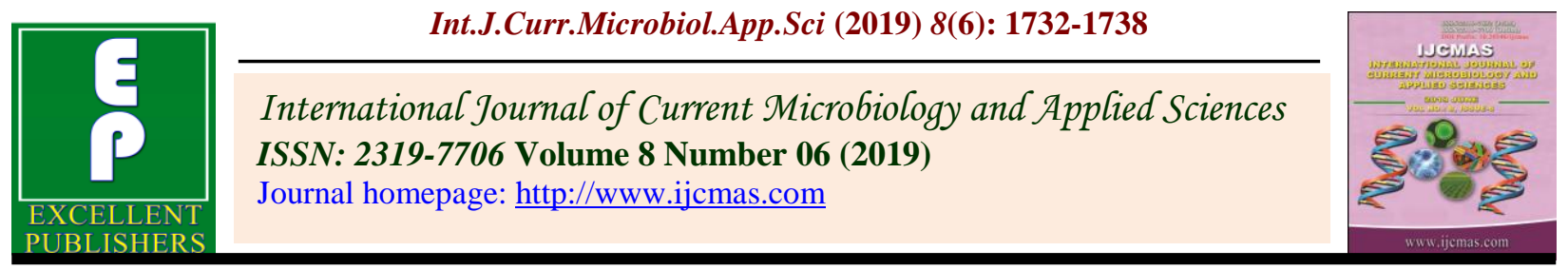

Original Research Article

https://doi.org/10.20546/ijcmas.2019.806.207

\title{
Extended Spectrum $\beta$-Lactamases and Metallo $\beta$-Lactamases Production in Klebsiella pneumoniae Isolates Causing Pneumonia in Rural Population of Uttar Pradesh, India
}

\author{
Nitisha Malik* , Dakshina Bisht and Sameer Singh Faujdar \\ Department of Microbiology, Santosh Medical College \& Hospital Ghaziabad, India \\ *Corresponding author
}

\begin{abstract}
A B S T R A C T
Keywords

Klebsiella pneumoniae, Extended Spectrum Beta-Lactamases (ESBL), Metallo BLactamases (MBL)

Article Info

Accepted:

15 May 2019

Available Online:

10 June 2019

The drug resistance among the gram negative bacteria is present as a serious global problem. ESBLs and MBLs are the important members of beta-lactamases produced mainly by gram negative bacteria and are responsible for mediating resistance to extendedspectrum cephalosporins and carbapenem. These enzymes are commonly detected in the members of the Enterobacteriaceae like Klebsiella pneumoniae and Escherichia coli. A cross-sectional study was conducted at Department of Microbiology, Santosh Medical College \& Hospital Ghaziabad by using K. pneumoniae isolated from sputum samples. Antimicrobial susceptibility testing was performed by Kirby-Bauer disc diffusion technique. ESBL and MBL production were detected by using phenotypic methods. Out of the 200 sputum samples, 72 samples were confirmed and identified as K. pneumoniae. Out of total 72 isolates, $48(66.6 \%)$ were found to be resistant to Ceftazidime, Ceftriaxone, Cefotaxime and $24(33 \%)$ isolates were found to be ESBL producers. On the other hand 40 $(55.5 \%)$ isolates were found to be resistant to imipenem and $16(22.2 \%)$ isolates were found to be MBL producers.
\end{abstract}

\section{Introduction}

The members of the Enterobacteriaceae family have an important role in nosocomial and community acquired infections. In recent years, $K$. pneumoniae have become important pathogens in various types of infections (Sunilkumar Biradar and Roopa, 2015). Klebsiella species is a Gram negative opportunistic nosocomial pathogen and is known to cause community acquired infections. Klebsiella is known to cause bacterial pneumonia, urinary tract infection, wound infections, blood infections and infections in the intensive care unit (Fish and Ohlinger, 2006). Antimicrobial resistance has become a serious public health problem worldwide. Infections caused by resistant bacteria are associated with increased morbidity and mortality than those caused by susceptible pathogens (Travers and Barza, 2002). Infections caused by resistant bacteria led to prolonged hospital stays, increased health care costs and in many cases to untreatable infections (Byarugaba, 2004). The incidence of infection due to extended- 
spectrum $\beta$-lactamase (ESBL) producing organisms has increased sharply especially in Escherichia coli and Klebsiella pneumoniae and also by Pseudomonas aeruginosa and Acinetobacter baumannii. The growing prevalence of ESBL producing organisms is responsible for increased use of carbapenems (Nordmann P et al., 2009). But extensive and sometimes unnecessary use of carbapenems has facilitated the emergence of carbapenem resistant bacteria by production of carbapenem hydrolysing enzyme, metallo- $\beta$ lactamase (MBL). MBL producing Pseudomonas and Acinetobacter isolates are common cause of nosocomial infections but other MBL producing gram negative bacteria like E. coli and Klebsiella spp. are also emerging as a public health concern worldwide (Manoj Kumar et al., 2015).

With this in mind, the present study was designed to increase awareness and demonstrate the need to detect and monitor the occurrence of novel-lactamases ESBLs and MBL. The goal was to investigate the occurrence (not prevalence) of these types of lactamases in our tertiary care hospital and to use the findings to alert health professionals about the need for vigilance and preparedness. Although these enzymes may be produced by a variety of organisms, the study focused only on Klebsiella pneumoniae because this species is the most common ESBL-producing organism and MBL production has also increased in over the period.

\section{Materials and Methods}

This study was conducted at Department of Microbiology, Santosh Medical College \& Hospital Ghaziabad. Fresh expectorated sputum samples were collected in a sterile wide mouth container. Patient was asked to rinse mouth thoroughly with water to reduce the amount of oral bacteria that are normally present that can contaminant the sputum.
Total of 200 sputum samples with clinical history of pneumonia were cultured on Blood agar and MacConkey agar and the isolates were identified by standard biochemical tests like Gram staining (Gram negative short and stout bacilli seen), Indole test (negative), Voges-Proskauer test (positive), Citrate utilization test (positive), Urease production test (positive), Triple sugar iron agar (A/A with gas), acid and abundant gas production from glucose, lactose, sucrose, maltose and mannitol sugar fermentation tests (K.N. Ravichitra et al., 2014).

\section{Antimicrobial susceptibility testing}

All Klebsiella pneumoniae isolates were screened for antimicrobial susceptibility testing by Kirby-Bauer disc diffusion method on Mueller-Hinton agar (Hi-Media) and interpreted as per CLSI guidelines (K. N. Ravichitra et al., 2014). A log phase broth culture inoculums of the isolate with a turbidity equivalent to McFarland 0.5 standard $\left(1.5 \times 10^{8} \mathrm{CFU} / \mathrm{ml}\right)$ was prepared and lawn cultured on the Mueller-Hinton agar and allowed to dry. Antibiotic discs were applied to the Mueller Hinton agar surface with the help of sterile forceps (Joshua T Freeman et al., 2014).

\section{ESBL detection tests}

\section{Screening test}

All $K$. pneumoniae isolates were screened for ESBLs by disc diffusion method. In the presumptive test to detect potential ESBL producers, all the isolates were screened for susceptibility to ceftazidime $(30 \mu \mathrm{g})$ and cefotaxime $(30 \mu \mathrm{g})$ antibiotic discs (Himedia, Mumbai). Results were interpreted based on the CLSI guidelines as follows: zones of inhibition of $\leq 22 \mathrm{~mm}$ for ceftazidime and $\leq 27 \mathrm{~mm}$ for cefotaxime indicated ESBL production. The less susceptible or resistant 
isolates were subjected to confirmatory test (CLSI, 2015).

\section{Confirmatory test}

The ESBL producing $K$. pneumoniae isolates were confirmed by CLSI phenotypic confirmatory test of combined disc assay method.15 One disc each of ceftazidime $(30 \mu \mathrm{g})$, Ceftriaxone $(30 \mu \mathrm{g})$ and cefotaxime $(30 \mu \mathrm{g})$ alone and one in combination with clavulanic acid $(10 \mu \mathrm{g})$ were placed at a distance of $20 \mathrm{~mm}$ on a Muller Hinton agar plate inoculated with a bacterial suspension of 0.5 McFarland turbidity standards, and incubated overnight at $37^{\circ} \mathrm{C}$. The ESBLproducing strains showed $\geq 5 \mathrm{~mm}$ increase in zone diameter for either antimicrobial agent tested in combination with clavulanic acid versus its zone when tested alone. E. coli ATCC 25922 was used as quality control strain (CLSI, 2015 and Tewari et al., 2018).

\section{MBL Detection tests}

\section{Screening test}

All K. pneumoniae isolates were screened for MBL production by testing their susceptibility to imipenem $(10 \mu \mathrm{g})$ antibiotic discs (Himedia, Mumbai) using Kirby Bauer disk diffusion method. All the isolates with an inhibition zone diameter of $\leq 19 \mathrm{~mm}$ were considered as screen positive for MBL and were subjected to confirmatory test (CLSI, 2015).

\section{Confirmatory test}

All screen positive $K$. pneumoniae isolates were confirmed for metallo- $\beta$-lactamase production. One disc of imipenem $(10 \mu \mathrm{g})$ alone and one in combination with EDTA $(750 \mu \mathrm{g} / \mathrm{mL})$ were placed at a distance of 20 mm on a Muller Hinton agar plate inoculated with a bacterial suspension of $0.5 \mathrm{McFarland}$ turbidity standards, and incubated overnight at $37^{\circ} \mathrm{C}$. The E. coli strain demonstrating a zone diameter $\geq 7 \mathrm{~mm}$ around the imipenem/EDTA disc compared to that of imipenem disc alone was considered to be positive for the presence of MBLs. E. coli ATCC 25922 were used as quality control strain (CLSI, 2015 and Khosravi Y et al., 2012).

\section{Results and Discussion}

Out of the 200 sputum samples, 72 samples were confirmed and identified as $K$. pneumoniae. Age wise distribution of $K$. pneumoniae among males \& females is shown in Table 1.

Among the 72 isolates of $K$. pneumoniae 52 $(72.2 \%)$ were from male patients and 20 (27.7\%) were from females. Maximum isolation was found in the age group of above 50 years and least isolation was found in the age group of 10-20 years.

In the present study maximum numbers of isolates were found to be sensitive to Amikacin (80.5\%), Gentamycin $(80.5 \%)$, CoTrimaxazole $(69.4 \%)$ and Piperacillin $(45 \%)$. Maximum number of isolates was found to be resistant to Ceftazidime (66.6\%), Cefpodoxime (75\%), Imipenem $(55.5 \%) \&$ Ofloxacin $(61.1 \%)$.

Out of total 72 isolates, $48(66.6 \%)$ were found to be resistant to Ceftazidime, Ceftriaxone, Cefotaxime and 24 (33\%) strains were found to be ESBL producers.

Out of 72 isolates, $40(55.5 \%)$ were found to be resistant to imipenem and 16 (22.2\%) strains were found to be MBL producers.

Beta lactams are commonly included in the empirical antibiotic regimens for treatment of infections caused by gram negative bacteria. Indiscriminate use of broad spectrum 
Cephalosporins has become one of the major reasons for the high production of extended spectrum beta lactamase by gram negative bacteria. Until recently, carbapenems were the choice for the therapeutic management of multidrug-resistant gram-negative bacterial infections. Currently, the spread of carbapenem-resistant bacteria has caused greater concern due to the limited choice in antibiotics for treating infection (Behera et al., 2008 and Palucha, 1999).
In our study isolation rate of $K$. pneumoniae was $36 \%$ from sputum samples, however study done by Kholy and Amani (2011) showed $32.6 \%$ isolation rate of Klebsiella pneumoniae. According to the study of Vemula Sarojamma (2011) the isolation rate of $K$. pneumoniae was $31.2 \%$ which is also in contrast to our results. Research done by $\mathrm{S}$ Baby Padmini (2004) revealed $14.11 \%$ isolation rate which is lower comparative to our study (Table 2).

Table.1 Age and sex wise distribution of $K$. pneumoniae

\begin{tabular}{|c|c|c|}
\hline Age & Male & Female \\
\hline $\mathbf{1 0 - 2 0}$ years & 4 & 0 \\
\hline $\mathbf{2 0 - 3 0}$ years & 12 & 2 \\
\hline 30-40 years & 8 & 6 \\
\hline 40-50 years & 10 & 4 \\
\hline >50 years & 18 & 8 \\
\hline Total & 52 & 20 \\
\hline
\end{tabular}

Table.2 Antibiotic sensitivity pattern of $K$. pneumoniae

\begin{tabular}{|c|c|}
\hline Antibiotics & Sensitive \\
\hline Amikacin & $80 \%$ \\
\hline Gentamycin & $80 \%$ \\
\hline Co-Trimaxazole & $69 \%$ \\
\hline Piperacillin & $45 \%$ \\
\hline Ceftazidime & $33 \%$ \\
\hline Ceftriaxone & $33 \%$ \\
\hline Cefotaxime & $33 \%$ \\
\hline Imipenem & $55 \%$ \\
\hline Ofloxacin & $39 \%$ \\
\hline Ciprofloxacin & $55 \%$ \\
\hline Tetracycline & $40 \%$ \\
\hline
\end{tabular}

\begin{tabular}{|l|c|c|}
\hline & $\begin{array}{c}\text { Ceftazidime/Ceftriaxone/Cefotaxime } \\
\text { Resistant }\end{array}$ & ESBL producers \\
\hline Klebsiella pneumoniae & $48(67 \%)$ & $24(33 \%)$ \\
\hline
\end{tabular}

Klebsiella pneumoniae

\section{Imipenem Resistant}

$40(55 \%)$
MBL producers $16(22 \%)$ 
The susceptibility pattern of $K$. pneumoniae observed in our study showed maximum sensitivity to Amikacin (80\%) and Gentamycin (80\%) followed by Cotrimaxazole (69\%), Ciprofloxacin (55\%), and Imipenem (55\%). Kholy and Amani (2011) reported susceptibility to Amikacin (66.5\%), Gentamycin (42\%), Co-Trimaxazole (24.3\%), and Ciprofloxacin (31\%) whereas Asati Rakesh Kumar (2013) reported susceptibility to Amikacin (93\%), Gentamycin (41\%), CoTrimaxazole (47\%) and Ciprofloxacin (51\%).

In the present study $K$. pneumoniae was found to be highly resistance to Ceftazidime (77\%), Ceftriaxone (77\%), Cefotaxime (77\%) followed by Ofloxacin (61\%) and Tetracycline (60\%). VemulaSarojamma (2011) in their study also reported high resistant to Ceftazidime (61\%), Amoxyclav (39), Imipenem (84\%), Amikacin (56\%), Gentamycin (40\%) whereas Asati Rakesh Kumar (2013) reported higher resistance to Tetracycline (85\%), Ceftizoxime (73\%), Cefuroxime (66\%). VemulaSarojamma (2011) also reported resistance to Ceftazidime (61\%), Kholy and Amani (2011) reported lower resistance to ceftazidime (25\%). I. Shukla (2004) reported higher ceftazidime resistance $(88.3 \%)$ compared to our study.

It is important for clinical microbiology laboratories to be able to detect ESBLs and carbapenem-hydrolyzing beta - lactamases because these enzymes are either associated with problems of false susceptibility or have significant infection control implications, or both. Extended-spectrum $\quad \beta$-lactamases (ESBLs) are frequently encountered among clinical Enterobacteriaceae, predominantly in E. coli and Klebsiella pneumoniae.

The present study showed much lower occurrence of ESBL and MBL producing Klebsiella pneumoniae, which was ranging from $30 \%$ to $75 \%$ reported from various parts of the world. Present study showed $33 \%$ ESBL production among Klebsiella pneumoniae isolates. Another study from U.S. (Ellen Smith Moland et al., 2002) revealed $75 \%$ occurrence of ESBL producer in Klebsiella pneumoniae isolates from 24 U.S. Hospitals. Kholy and Amani (2011) have shown that $64 \% \mathrm{~K}$. pneumoniae isolates were ESBL producers, Baby Padmini (2004) have reported $40 \%$ ESBL producers which were at higher side compared to present study Whereas I. Shukla (2004) have shown that $30 \% \mathrm{~K}$. pneumoniae isolates were ESBL producers and Singh P (2018) reported only $6.7 \% \mathrm{~K}$. pneumoniae isolates were positive for ESBL production in his research.

In our study $22 \%$ Klebsiella pneumoniae isolates were $\mathrm{MBL}$ producers Whereas $\mathrm{S}$. Nagaraj (2012) reported $75 \%$ of MBL production While in a study done by Giske CG (2011) showed $69.4 \%$ of MBL production rate in $K$. pneumoniae isolates causing pneumonia.

Antibiotic overuse is an important contributor for the emergence and spread of resistance. Since last 15years, acquired resistance which is mainly mediated by ESBLs and MBLs has been increasingly reported worldwide including India not only in $\mathrm{K}$. pneumoniae, but also among members of Enterobacteriaceae.

Detection of ESBL and carbapenemases in Enterobacteriaceae is still very important for infection control and epidemiological point of view, and may impact greatly on patient management.

\section{Acknowledgment}

I humbly thank to Dr. DakshinaBisht (Professor and Head, Department of Microbiology) for technical guidance and support during my study period. 


\section{References}

Asati Rakesh Kumar (2013). Antimicrobial Sensitivity Pattern of Klebsiella Pneumoniae isolated from Sputum from Tertiary Care Hospital, Surendranagar, Gujarat and Issues Related to the Rational Selection of Antimicrobials. Sch. J. App. Med. Sci.; 1(6): 928-933.

Behera B, Mathur P, Das A, Kapil A, Sharma V. (2008). An evaluation of four different phenotypic techniques for detection of metallo- $\beta$-lactamase producing Pseudomonas aeruginosa; 26(3): 233-237.

Byarugaba DK (2004). A view on antimicrobial resistance in developing countries and responsible risk factors. Int J Antimicrob Agents; 24(2): 105-

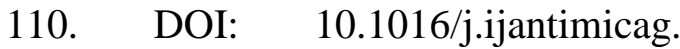
2004.02.015

Clinical Laboratory Standard Institute (2015). Performance standards for antimicrobial susceptibility testing; Twenty-Fifth informational supplement document. Wayne, PA: CLSI; 35: M100-S25.

El KholyAmani A., Howayda E. Gomma, Mariam A. Younan, Eman H. Thabet, Mona M.A. Haleim, Mervat G. El Ananya and Ahmed A.S. Hendey (2011). Extended spectrum b-lactamaseproducing Klebsiella pneumoniae and Escherichia coli strains in a pediatric teaching hospital in Egypt. Medical Research Journal; 10: 27-31. DOI: 10.1097/01.MJX.0000397210.93550.d2 Ellen Smith Moland, Jennifer A. Black, Jason Ourada, Mark D. Reisbig, Nancy D. Hanson, and Kenneth SThomson (2002). Occurrence of Newer Lactamases in Klebsiella pneumoniae Isolates from 24 U.S. Hospitals. Antimicrobial Agents and Chemotherapy; 3837-3842. DOI: 10.1128/AAC.46.12.3837-3842.2002

Fish DN, Ohlinger MJ (2006). Antimicrobial resistance: factors and outcomes. Crit Care Clin; 22(2): 291-311.DOI: 10.1016/j.ccc.2006.02.006

Giske CG, Gezelius L, Samuelsen Ø, Warner M, Sundsfjord A, Woodford N.(2011). A sensitive and specific phenotypic assay for detection of metallo- $\beta$ lactamases and KPC in Klebsiella pneumoniae with the use of meropenem disks supplemented with aminophenylboronic acid, dipicolinic acid and cloxacillin. ClinMicrobiol Infect.; 17(4):552-6.doi: 10.1111/j.1469-0691.2010.03294.x.

Joshua $\mathrm{T}$ Freeman, Jessica Nimmo, Eva Gregory, Audrey Tiong, Mary De Almeida, Gary N McAuliffe and Sally A Roberts (2014). Predictors of hospital surface contamination with Extendedspectrum Beta-lactamase-producing Escherichia coli and Klebsiella pneumonia: patient and organism factors. Antimicrobial Resistance and Infection Control; 3(5):1-7.

K.N. Ravichitra, P.Hema Prakash, S. Subbarayudu and U. Sreenivasa Rao (2014). Isolation and antibiotic sensitivity of Klebsiella pneumonia from pus, sputum and urine samples. Int. J. Curr. Microbiol. App. Sci; 3(3): 115-119.

Khosravi Y, Loke MF, Chua EG, Tay ST, Vadivelu J (2012). Phenotypic detection of metallo- $\beta$-lactamase in imipenemresistant Pseudomonas aeruginosa. Scientific World Journal; 2012: 654939. doi: 10.1100/2012/654939

Manoj Kumar, Renu Dutta, SonalSaxena, SmitaSinghal (2015). Risk Factor Analysis in Clinical Isolates of ESBL and MBL (Including NDM-1) Producing Escherichia coli and Klebsiella Species in a Tertiary Care Hospital. Journal of Clinical and Diagnostic Research; Vol-9(11): DC08DC13. DOI: 10.7860/JCDR/2015/ 
15672.6766

Nordmann P, Cuzon G, Nass T (2009). The real threat of Klebsiella pneumoniae carbapenemase-producing bacteria. Lancet Infect Dis.; 9:228-36.doi: 10.1016/S1473-3099(09)70054-4.

Palucha A, Mikiewiez B, Hryniewiez W, Griadkowski M (1999). Concurrent outbreaks of extended spectrum lactamase producing organisms of the family Enterobacteriaceae in a Warsaw Hospital. J Antimicrob Chemother; 44(4): 489-499.

S Baby padmini, Appalaraju B (2004). Extended spectrum -lactamases in urinary isolates of Escherichia coli and Klebsiella pneumoniae - Prevalence and susceptibility pattern in a tertiary care hospital. Indian J Med Microbiol; 22(3): 172-4.

S Nagaraj, SP Chandran, P Shamanna, R Macaden (2012). Carbapenem resistance among Escherichia coli and Klebsiella pneumoniae in a tertiary care hospital in south India. Indian $\mathbf{J}$ Med Microbiol; 30: 93-5.

Shukla I., R Tiwari, M Agrawal (2004). Prevalence of extended spectrum lactamase producing Klebsiella pneumoniae in a tertiary care hospital. Indian J Med Microbiol; 22: 87-91.
Singh P (2018). The outcome of different $\beta$ lactamase production in Klebsiella pneumoniae subspecies pneumoniae isolated from different clinical specimen in tertiary care hospital: a study. Int $\mathbf{J}$ Res Med Sci; 6: 1568-73.

SunilkumarBiradar and C. Roopa (2015). Isolation and Antibiogram of Klebsiella species from Various Clinical Specimens. Int.J.Curr.Microbiol.App. Sci; 4(9): 991-995.

Tewari R, Mitra SD, Ganaie F, Venugopal N, Das S, Shome R, et al., (2018). Prevalence of extended spectrum $\beta$ lactamase, AmpC $\beta$-lactamase and metallo $\beta$-lactamase mediated resistance in Escherichia coli from diagnostic and tertiary healthcare centers in south Bangalore, India. Int J Res Med Sci; 6:1308-13. DOI: http://dx.doi.org/ 10.18203/2320-6012.ijrms20181288

Travers K, Barza M (2002). Morbidity of infections caused by antimicrobialresistant bacteria.Clin Infect Dis; 34(3): S131-4.DOI: 10.1086/340251

Vemula Sarojamma, Vadde Ramakrishna (2011). Prevalence of ESBL-Producing Klebsiella pneumoniae Isolates in Tertiary Care Hospital. ISRN Microbiology; Article ID 318348, 1-5. doi.org/10.5402/2011/318348

\section{How to cite this article:}

Nitisha Malik, Dakshina Bisht and Sameer Singh Faujdar. 2019. Extended Spectrum $\beta$ Lactamases and Metallo $\beta$-Lactamases Production in Klebsiella pneumoniae Isolates Causing Pneumonia in Rural Population of Uttar Pradesh. Int.J.Curr.Microbiol.App.Sci. 8(06): 17321738. doi: https://doi.org/10.20546/ijcmas.2019.806.207 